\title{
MiR-301a-5p/SCIN promotes gastric cancer progression via regulating STAT3 and NF-KB signaling
}

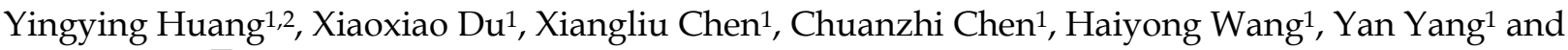 \\ Lisong Teng $1{ }^{\bowtie}$ \\ 1. Department of oncological surgery, The First Affiliated Hospital, College of Medicine, Zhejiang University. \\ 2. Cancer Institute (Key Laboratory for Cancer Intervention and Prevention, China National Ministry of Education, Zhejiang Provincial Key Laboratory of \\ Molecular Biology in Medical Sciences), The Second Affiliated Hospital, Zhejiang University School of Medicine, China. \\ $\triangle$ Corresponding author: Lisong Teng, Department of oncological surgery, The First Affiliated Hospital, College of Medicine, Zhejiang University, Hangzhou, \\ Zhejiang province. Tel/Fax: 0571-87236734; Email: 1steng@zju.edu.cn
}

(c) The author(s). This is an open access article distributed under the terms of the Creative Commons Attribution License (https://creativecommons.org/licenses/by/4.0/). See http://ivyspring.com/terms for full terms and conditions.

Received: 2021.02.24; Accepted: 2021.06.29; Published: 2021.07.06

\begin{abstract}
Objective: Gastric cancer (GC) is a type of highly malignant cancer. Although the diagnostic and therapeutic methods are innovating, the outcome of GC patients is still poor. Therefore, our research was carried out to explore potential molecular mechanism in the diagnosis of GC.

Materials and methods: Bioinformatics analyses were used to obtain microRNA and target mRNA of interest. The expression level of miR-30la-5p and Scinderin (SCIN) mRNA were detected by quantitative real-time PCR (qRT-PCR). Western blot assay was used to investigate SCIN protein level. Cell Counting Kit-8 assay (CCK-8) and colony formation assay were used to investigate cell proliferation ability. Transwell assay was employed to examine cell motility. The interaction between miR-301a-5p and SCIN mRNA was verified by dual-luciferase reporter assay.

Results: The qRT-PCR analysis revealed that the expression of miR-301a-5p was higher in gastric cancer tissues than para-cancer tissues $(\mathrm{P}<0.05)$. Cox regression analysis showed upregulated miR-301a-5p was associated with larger tumor size $(P=0.036)$ and more advanced TNM stage $(P=0.048)$. The Kaplan-Meier analysis showed a correlation between increased miR-301a-5p expression and shorter overall survival $(\mathrm{OS})(\mathrm{P}=0.018)$. By using bioinformatic analysis, $\mathrm{SCIN}$ was predicted as one of the targets of miR-30la-5p. Overexpressing miR-30la-5p promoted proliferation and motility of GC cells while knockdown of SCIN exhibited the same performance. Further, we verified the alteration of miR-301a-5p and SCIN expression level could affect the epithelial-mesenchymal transition (EMT) progression on GC cells via STAT3 and NF-KB signaling.

Conclusion: Highly expressed miR-301a-5p was associated with aggressiveness of GC. Upregulation of miR-301a-5p promoted malignant phenotype of GC by targeting SCIN. The present results indicated miR-301a-5p might be a promising molecule in the prognosis of GC.
\end{abstract}

Key words: MicroRNA, miR-301a-5p, gastric cancer (GC), progression, EMT

\section{Introduction}

Gastric cancer (GC) is the fifth most common cancer with high cancer-related mortality around the world[1]. The mortality of gastric cancer among cancer-related death has been the second place in China[2]. Although there are various treatments for gastric cancer, the five-year survival rate of patients with advanced GC is still frustrating[3]. Thus, further studies on GC pathogenesis and identifying predictive biomarkers of GC patients are in at imminent.

MicroRNAs(miRNAs) is a type of non-coding RNA with about 22 nucleotides which negatively regulate gene expression by interacting with the 3 -untranslated regions (3'-UTR) of target mRNAs and suppressing translation or inducing degradation[4]. It has been reported that microRNAs 
displayed pleiotropic functions in cellular progresses involved in carcinogenesis and a large number of researches showed that aberrantly expressed microRNAs played diversified roles in various tumors[5, 6]. MiR-301a-5p is regarded as an important member of miRNAs family in studies[7, 8]. Overexpressed miR-301a-5p could rescue the depression in proliferation, migration and invasion of hepatocellular carcinoma (HCC) cell which were induced by down-regulation of lncRNA EPB41L4B-AS2[7]. Hypoxic tumor-derived exosomal miR-301a regulated M2 macrophage polarization and further promoted pancreatic cancer metastasis by PTEN/PI3KY pathway[9]. In another study, miR-301a acted as NF-kB activator in pancreatic tumor cell[10]. Upregulated expression of miR-301a in prostate cancer and breast cancer tissues indicated poor prognosis[11, 12]. Also, miR-301a played a role in reducing intestinal epithelial integrity and promoting intestinal inflammation, thus leading to colitis-associated cancer development by depressing BTG1 expression[13].

MicroRNAs modulate cellular progresses by regulating targeted genes. Scinderin (SCIN), a type of $\mathrm{Ca}^{2+}$-dependent actin-severing and -capping protein, is required for megakaryocyte differentiation, maturation, polyploidization and apoptosis with the release of platelet-like particles[14]. Another study found that SCIN together with Cdc42 performed important function in the migration and invasion of melanoma cell[15]. Human bladder cancer cell line HT1376 with high SCIN expression showed significant cisplatin resistant and SCIN mRNA knockdown reduced cell proliferation with mitochondriamediated apoptosis[16]. Therefore, SCIN is important for regulating cancer cell survival and migration.

In the present study, we investigated the relationship between miR-301a-5p and SCIN and their regulatory effects via STAT3 and NF-kB signaling on the proliferation and motility of gastric cancer cell lines. These results can help to explore novel biomarkers and therapeutic approaches to GC.

\section{Materials and methods}

\section{Patients and samples}

Gastric cancer tissue and para-cancer tissue samples were randomly collected from 45 patients undergoing gastric cancer surgery between 2010 and 2015 in the First Affiliated Hospital of Zhejiang University, Hangzhou, Zhejiang Province, China. All patients had not received radiotherapy or chemotherapy before radical gastrectomy and follow-up information was available for all patients. All the samples were pathologically diagnosed as gastric cancer. Patients were informed that specimens were stored by the hospital and used for scientific research potentially. Patients signed informed consent to participants was exempted by the Clinical Research Ethics Committee of the First Affiliated Hospital of Zhejiang University (Approval No:2020-766). The study was conducted in accordance with Declaration of Helsinki and its amendments.

\section{Bioinformatics Analysis}

The miRNAs and mRNAs expression profiles of stomach adenocarcinoma (STAD) were firstly downloaded from GEO Database (http://www .ncbi.nlm.nih.gov/geo/), and the differential analysis was performed, the threshold was set as absolute value of $\log 2$ fold change $>1$, adjusted $p$ value $<0.05$. The miRNAs expression level of gastric cancer and normal gastric tissues was reconfirmed by TCGA data (http://www.cancer.gov/tcga). Target genes of miRNA were predicted in TargetScan (http:// www.targetscan.org/), and the final target gene was determined by intersecting with differential expression mRNAs.

\section{Cell culture and transfection}

GC cell lines AGS, HGC-27, MGC803, SGC7901, BGC823, SNU-1, NCI-N87, MKN-45 were purchased from the Cell Bank of the Chinese Academy of Sciences (Shanghai, China) and human non-malignant gastric mucosal cell line GES-1 was obtained from the China Center for Type Culture Collection (CCTCC). All the cell lines were cultured according to their protocols: AGS, HGC-27, MGC803, SGC7901, BGC823, SNU-1, NCI-N87, MKN45 were cultured in RPMI1640 medium with 10\% fetal bovine serum sand $100 \mathrm{u} / \mathrm{ml}$ penicillin/streptomycin, GES-1in DMEM medium with $10 \%$ fetal bovine serum and $100 \mathrm{u} / \mathrm{ml}$ penicillin/streptomycin in cell incubator with 5\% $\mathrm{CO} 2$ at $37^{\circ} \mathrm{C}$.

Cells were transfected with $50 \mathrm{nM}$ miRNA mimics or $75 \mathrm{nM}$ inhibitor using lipofectamine 3000 (Invitrogen, USA). Interfering RNAs (siRNA) targeting SCIN was obtained from Genepharma (Shanghai, China) (siRNA1: 5'-GGUGAGAGCCAC AGAAGUUTT-3'; siRNA2: 5'-GGAGCAGAGUAUG UAGCAATT-3'; siRNA3: 5'-GGACACCAAUUGUCA UCAUTT-3') and transfected at concentration of 50nM. Random sequence of RNA duplex was used as negative control for miRNA and siRNA. Plasmids expressing sh-SCIN or sh-NC were purchased from Genepharma (Shanghai, China).

\section{Quantitative real-time polymerase chain reaction (qRT-PCR)}

Total RNA was extracted from cells and tissues by using Trizol (Invitrogen) followed by RT-PCR 
reaction. MiR-301a-5p was reverse-transcribed using Mir-X miRNA First-Strand Synthesis Kit (Clontech, Osaka, Japan) and measured using TB Green ${ }^{\circledR}$ Premix Ex Taq ${ }^{\mathrm{TM}}$ II (Takara, Osaka, Japan) via StepOne Real-Time PCR System (Applied Biosystems, Foster City, CA, USA). U6 RNA was used as miRNAs endogenous control and target gene mRNA level was normalized to GAPDH expression. MiR-301a-5p and SCIN specific primers were purchased from Tsingke (miR-301a-5p GCTCTGACTTTATTGCACTACT; SCIN FW 5'-ATGGCTTCGGGAAAGTTTATGT-3', SCIN RW 5'-CATCCACCATATTGTGCTGGG-3'). Relative expression analysis was performed using the comparative CT method $\left(2^{-\Delta \Delta C T}\right)$.

\section{Dual-luciferase reporter assay}

To verify the target sites of miR-301a-5p and SCIN, HEK-293T cell were co-transfected with miR-301a-5p mimics or control and luciferase reporter constructs containing wild-type or mutated SCIN 3'-UTR. Cells were split and luciferase activity was measured using Dual-Luciferase Reporter Assay Kit (Promega, Madison, WI, USA).

\section{Western blotting assay}

Total proteins were extracted from GC cells and tissues, and their concentrations were measured by Pierce $^{\mathrm{TM}}$ Rapid Gold BCA Protein Assay Kit (Thermo Fisher Scientific). Equal amounts of total protein sample from various samples were separated by SDS-PAGE and transferred onto PVDF (polyvinylidene fluoride) membrane (Millipore, USA). The specific primary antibodies used in western blotting assay were as follow: SCIN (Abcam, \#191396); E-Cadherin (CST, \#3195S); N-cadherin (Abcam, \#ab76011); ZEB1 (Abcam, \#ab203829); phospho-IкBa (Ser32/36) (CST, \#9246), ІкBa (Abcam, \#32518); SNAI1 (CST, \#3879); STAT3 (Abcam, \#68153); phospho-STAT3 (S727) (Abcam, \#131103); NF-kB p65 (Abcam, \#32536); NF-kB p65 (phospho-S536) (Abcam, \#76302); GAPDH (CST, \#3683).

\section{Cell growth assay}

Transfected cells and control cells were seeded in 96-well plates at a density of $1 \times 10^{3}$ cells/well and incubated at $37^{\circ} \mathrm{C}$. Cell viability was analyzed at $6 \mathrm{~h}$, 24h, $48 \mathrm{~h}$ and $72 \mathrm{~h}$ using Cell Counting Kit-8 (CCK-8, Dojindo, Kumamoto, Japan) according to the manufacturer's protocol.

\section{Cell migration and Transwell assay}

Transwell ( $8 \mu \mathrm{m}$ with 24 cluster Plate) (Corning, USA) were coated with $50 \mu \mathrm{L}$ of Matrigel (BD, USA)/PRMI-1640 medium (1:14, v/v) for $30 \mathrm{~min}$ at $37^{\circ} \mathrm{C}$. A total of $2 \times 10^{4}$ cells were seeded into the upper chambers with $100 \mu \mathrm{L}$ serum-free RPMI-1640 medium while the lower chambers were filled with $700 \mu \mathrm{L}$ RPMI-1640 medium containing 10\%FBS. After $48 \mathrm{~h}$ incubation, the chamber membranes were fixed with $4 \%$ paraformaldehyde for $15 \mathrm{~min}$. Cells on the upper surface of chamber membranes were removed with cotton swabs, and the cells on the lower surface of membranes were stained with crystal violet dye (Beyotime, China). The number of invading cells were counted in 5 random visual field and photographed under microscopic.

\section{Statistical analysis}

Data are presented as the mean tstandard deviation (SD) from at least three independent experiments. Student's $t$ tests were performed to analyze the differences between two groups and the Pearson $\chi^{2}$ tests were used for binary analysis by using IBM SPSS Statistics software, version 21. P values $<0.05$ were considered statistically significant.

\section{Results}

\section{Expression level of $\mathrm{miR}-301 \mathrm{a}-5 \mathrm{p}$ is upregulated in gastric cancer tissues and cell lines.}

We obtained 2 miRNAs expression profiles from GEO database (GSE78091 and GSE93415) and found that miR-301a-5p expression level was altered in GC $(|\log \mathrm{FC}|>1$, adjusted P value<0.05) (Figure 1a). Then, we reconfirmed the miR-301a-5p level of gastric cancer tissue and normal gastric tissue from TCGA-STAD data, it was upregulated in GC (Figure $1 b)$. Also, the level of miR-301a-5p in GC cell lines was determined, using GES-1 (a type of human gastric mucosal cell) as comparison (Figure 1c). Furthermore, we obtained 45 pairs GC patient tissues and performed qRT-PCR to measure the expression state of miR-301a-5p (Figure 1d). Table 1 listed the clinical characteristics of 45 patients and their relationships with miR-301a-5p expression. High expression of miR-301a-5p was significantly associated with larger tumor size $(\mathrm{P}=0.036)$ and more advanced TNM stage $(\mathrm{P}=0.048)$, but had no significant correlation with age and gender (Table 1). The correlation between miR-301a-5p expression and overall survival was analyzed by Kaplan-Meier analysis. The results showed that patients with increased expression of miR-301a-5p had shorter OS than patients with low level of miR-301a-5p ( $\mathrm{P}=0.018$ by log rank) (Figure 1e). The above results indicated miR-301-5p was elevated in GC tissues and GC cell lines and was associated with poor prognosis.

\section{Upregulation of miR-301a-5p promotes proliferation, migration and invasion in vitro.}

The functional roles of miR-301a-5p in the 
proliferation, migration and invasion of GC cells, which had important impact on tumorigenesis and metastasis, were investigated. We examined the effects of miR-301a-5p overexpression on proliferation and metastasis on AGS and MGC803 cell lines. The results of CCK-8 and colony formation assay showed that when transfecting cells with mimics of miR-301a-5p, cell viability was significantly increased and cell growth was promoted (Figure 2a, b). Meanwhile, the migration and invasion ability were increased (Figure 2c). We also transfected cells with inhibitors of miR-301a-5p to restrain the function of miR-301a-5p. As results, cell viability, proliferation, migration and invasion ability were reduced (Figure 2d-f). Additionally, expression of EMT-related proteins altered when miR-301a-5p level changed (Figure 2g, h). These results indicated that miR-301a-5p as an oncogene could promote proliferation, migration and invasion of GC cells.

Table 1: Correlation between hsa-mir-30la-5p expression and clinical features of the GC patients

\begin{tabular}{llll}
\hline \multirow{2}{*}{ Clinical parameter } & \multicolumn{2}{l}{ hsa-mir-301a-5p expression } & P value \\
\cline { 2 - 3 } & high & low & \\
\hline Age (years) & 11 & 12 & 0.436 \\
$\geq 60$ & 8 & 14 & \\
$<60$ & & & 0.478 \\
Gender & 12 & 19 & \\
Male & 7 & 7 & \\
Female & & & $\mathbf{0 . 0 3 6}$ \\
Tumor size & 11 & 7 & \\
$\geq 5 \mathrm{~cm}$ & &
\end{tabular}

\begin{tabular}{llll}
\hline Clinical parameter & hsa-mir-301a-5p expression & P value \\
\cline { 2 - 3 } & high & low & \\
\hline$<5 \mathrm{~cm}$ & 8 & 19 & $\mathbf{0 . 0 4 8}$ \\
TNM stage & & & \\
I-II & 4 & 13 & \\
III-IV & 15 & 13 & \\
\hline
\end{tabular}

Notes: *Statistical analyses by Pearson's $\chi 2$ test or Fisher's exact test Abbreviation: gastric cancer: GC

\section{MiR-30 la-5p targets SCIN and SCIN is downregulated in GC tissues and cell lines.}

Since miRNAs modulating cellular progresses by targeting downstream genes, we explored the target genes of miR-301a-5p. Online miRNAs analysis software (TargetScan) was used to predict the downstream mRNA of miR-301a-5p. Otherwise, we downloaded 2 mRNA expression profiles (GSE33651 and GSE54129) from GEO database and gained a gene set of downregulated genes with threshold of $|\log \mathrm{FC}|>1$ and adjusted $\mathrm{P}$ value $<0.05$. The expression sets and the predicted target gene set were intersected to obtain SCIN as interested targeted gene (Figure 3a). SCIN is a type of $\mathrm{Ca}^{2+}$-dependent actin-severing and -capping protein and mainly located in cytoplasm and cytoskeleton structure[17]. Then, we verified the SCIN expression level based on TCGA-STAD database (Figure 3b), qRT-PCR and western blot assay results of cell lines showed that SCIN expressed at low level in AGS and HGC-27, but comparative high level in MGC803 (Figure 3c-d). 45 pairs of GC cancer tissue and para-cancer tissue samples were examined, the
$\Lambda$

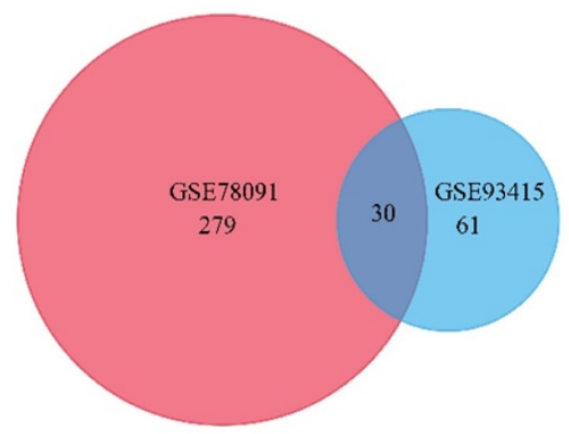

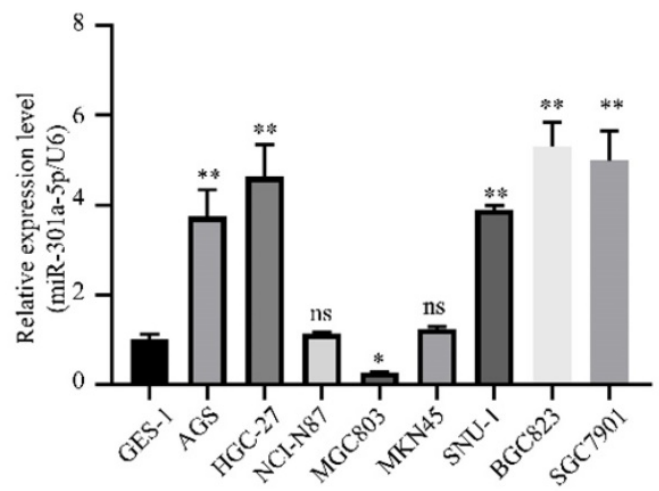
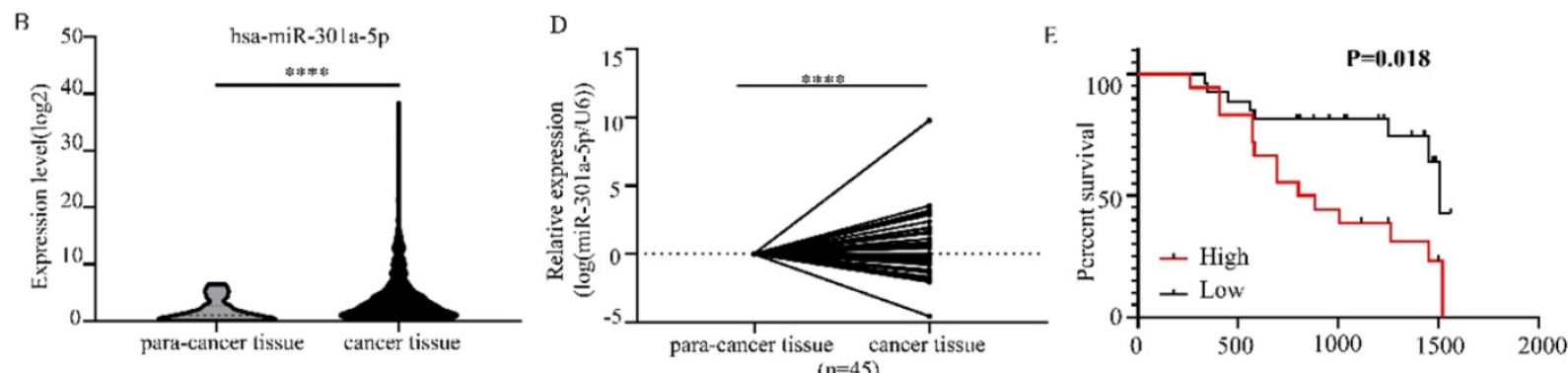

Figure 1: Expression level of miR-301 a-5p is upregulated in GC tissues and cell lines. (a) MiR-301a-5p was selected as target miRNA from GEO database and its expression level was altered in GC. (b-d) Expression of miR-301a-5p was increased in GC through verification of TCGA database, GC cell lines and GC samples. U6 was used as an internal control. (e) Patients with high miR-301a-5p expression had shorter overall survival $(O S)(P=0.018)$. * $p<0.05, * * p<0.01, * * * p<0.001, * * * * p<0.0001$. 
SCIN mRNA level was significantly down-regulated in tissues of GC patients (Figure 3e). Meanwhile, the expression level of SCIN protein extracted from GC patients was tested (typical pairs of tissues were showed) (Figure 3f). Further, we transfected MGC803 and AGS with mimics of miR-301a-5p and measured SCIN expression status by qRT-PCR and western blot, the miRNA level was sharply elevated while SCIN mRNA and protein expression were reduced (Figure $3 g$ ). Inversely, miRNA level was reduced and SCIN increased at the present of inhibitor of miR-301a-5p (Figure 3h). To further prove that miR-301a-5p works by targeting the $3^{\prime}$-UTR of SCIN mRNA, dual-luciferase reporter assay was performed. And results demonstrated that there was an acting site between miR-301a-5p and 3'-UTR of SCIN mRNA (Figure $3 \mathrm{i}$ ). The above results showed SCIN was the target of miR-301a-5p and its expression level was affected by miR-301a-5p.
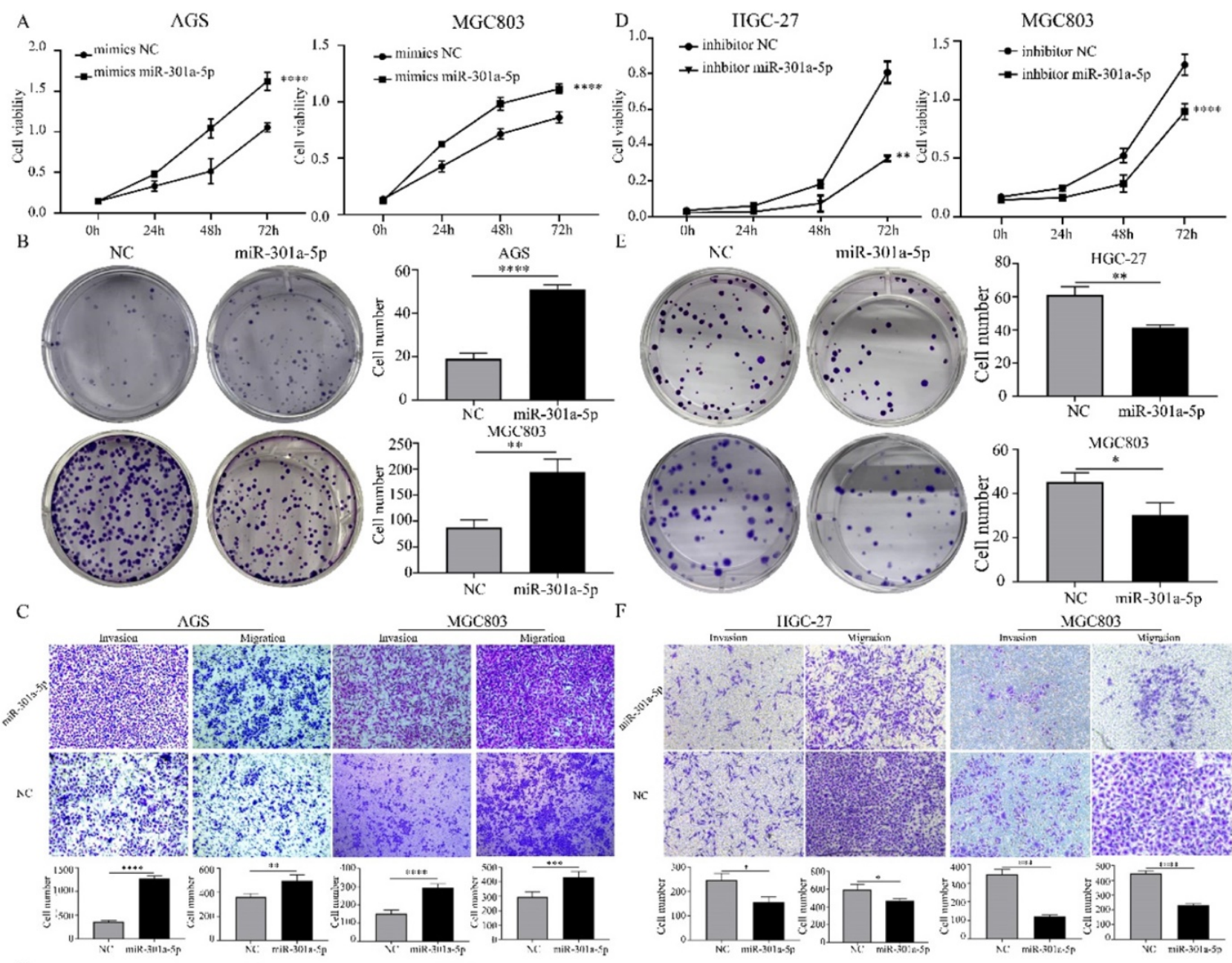

$\Lambda \mathrm{GS}$ MGC803
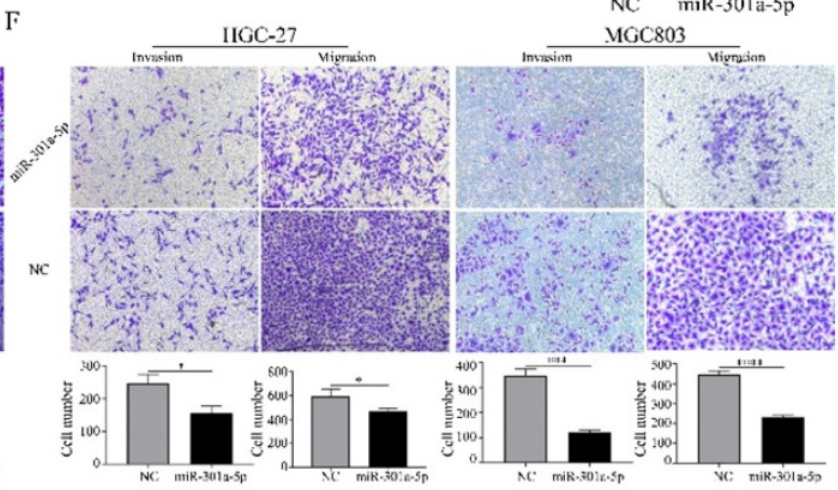

G
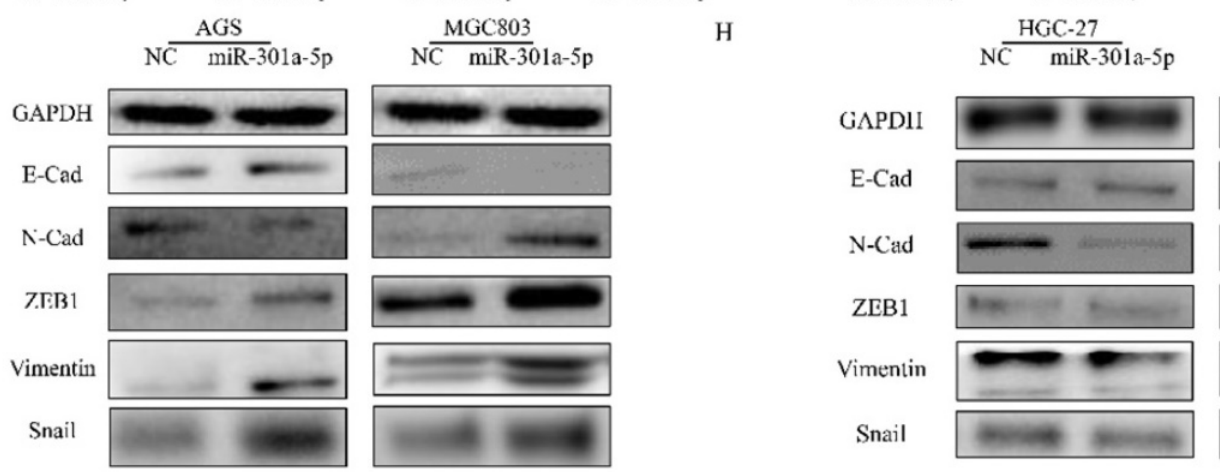

$\frac{\mathrm{MGC} 803}{\mathrm{NC} \quad \operatorname{miR}-301 \mathrm{a}-5 \mathrm{p}}$

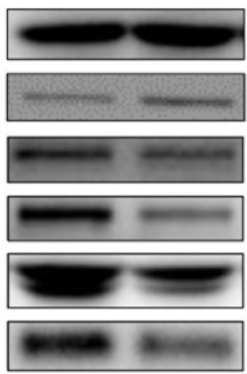

Figure 2: Increasing of miR-301a-5p promotes proliferation, migration and invasion in vitro, vice versa. (a-b) Cell proliferation was determined by CCK-8 and colony formation assay after transfection with mimics of miR-301a-5p. (c) Cell migration and invasion were assessed by Transwell assay after transfection with mimics of miR-301a-5p (magnification, 40x). (d-e) Cell proliferation was determined by CCK-8 and colony formation assays after transfection with inhibitor of miR-30la-5p. (f) Cell migration and invasion were assessed by Transwell assays after transfection with inhibitor of miR-301a-5p (magnification, $40 \times$ ). ( $g$-h) EMT markers of cell lines were measured after transfection with mimics and inhibitor of miR-301a-5p. * $p<0.05$, ** $p<0.01$, *** $p<0.001$, **** $p<0.0001$. 

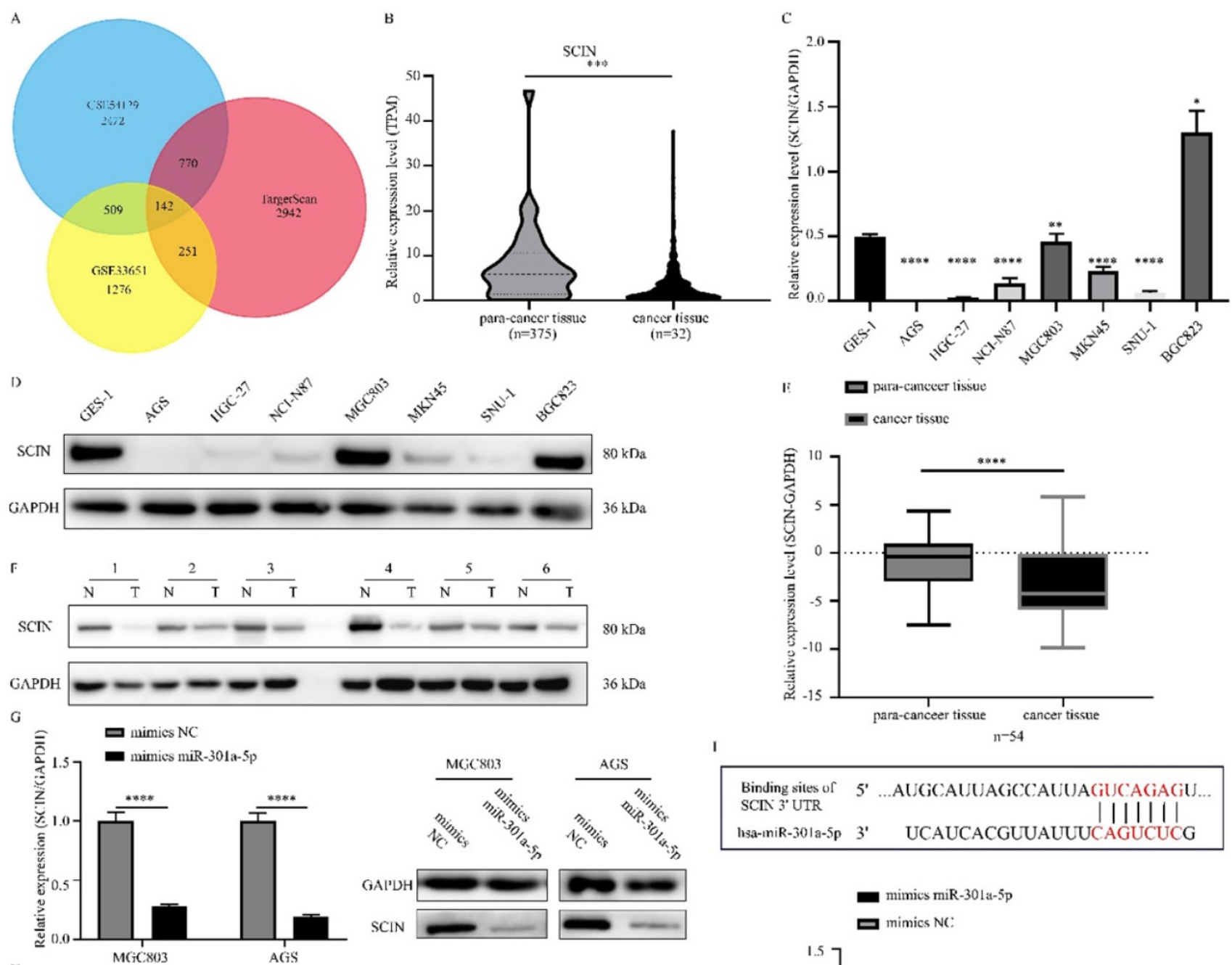

I

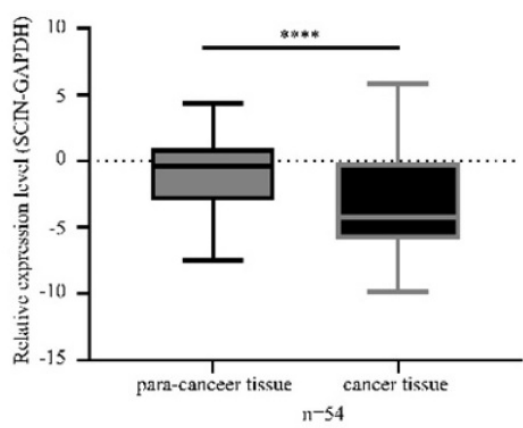

Binding sites of 5' ...AUGC.AUUAGCCAUUACiUCAGAGU. SCIN 3. UTR lisa-iniR-30la-5p 3' UCAUCACGUUAUUUCAGUCUCG
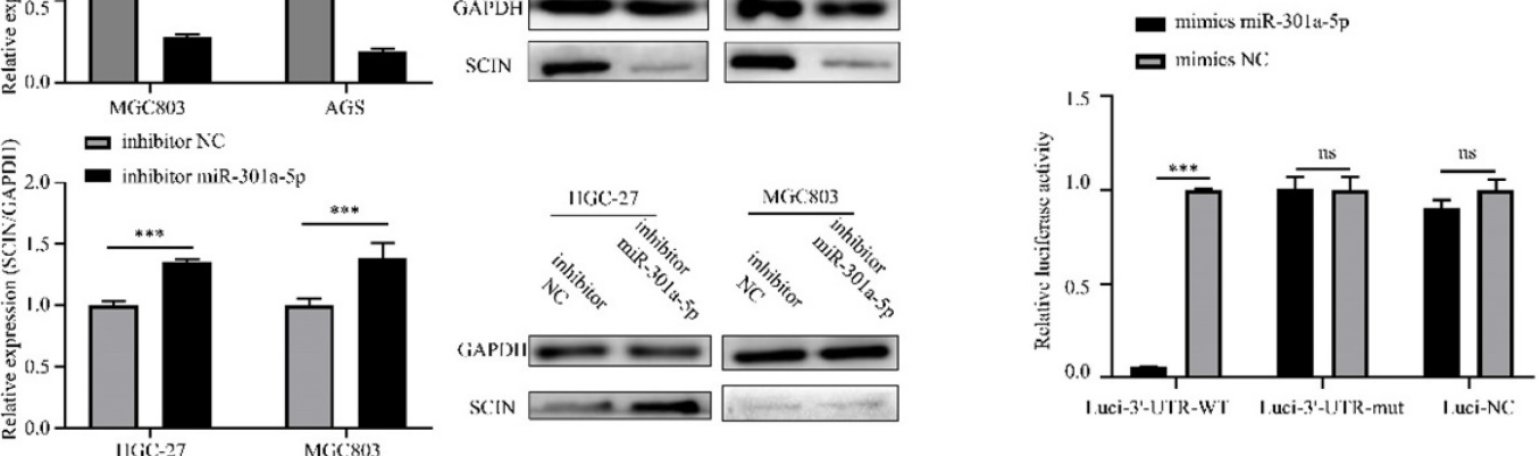

Figure 3: Direct regulation of SCIN by miR-301a-5p in gastric cancer. (a) SCIN was predicted as target as miR-301a-5p via bioinformatic analyses. (b) Expression level of SCIN in TCGA database. (c-d) mRNA and protein expression level of SCIN in GC cell lines by qPCR and western blot. GAPDH was used as internal control. (e-f) SCIN mRNA and protein level of GC patient sample was determined by $q R C R$ and western blot. (g) After transfecting mimics of miR-30la-5p in cell lines, the alteration of SCIN mRNA and protein were measured by QPCR and western blot. GAPDH was used as internal control. (h) After transfecting inhibitor of miR-301a-5p in cell lines, the alteration of SCIN mRNA and protein were measured by qPCR and western blot. GAPDH was used as internal control. (i) miR-301a-5p binding sites in the 3'-UTR of SCIN mRNA. Dual luciferase reports assays exhibited that miR-301a-5p markedly suppressed luciferase activity of HEK-293T carrying wild-type reporter constructs. $* p<0.05, * * p<0.01, * * * p<0.001, * * * * p<0.0001$.

\section{SCIN acts as tumor suppressor in GC.}

In order to further investigate the function of SCIN, we induced SCIN overexpression and knockdown in GC cell lines. Firstly, we transfected GC cell lines with siRNA to depress the expression of SCIN, qRT-PCR and western blot were used to verify the knockdown efficiency of siRNAs (Figure 4a-b). SCIN-depleted cells exhibited significant decreased proliferation, colony formation, migration and invasion abilities (Figure 4c-e). Furthermore, we transfected GC cells with overexpression plasmid and the efficiency of transfection was confirmed by western blot assay (Figure 5a). Conversely, overexpression of SCIN led to opposite results on cell proliferation, colony formation and ability of migration and invasion (Figure $5 b-d$ ). We also observed the level of EMT-related markers has changed with the alteration of SCIN. SCIN-knockdown group showed an increasing of mesenchymal markers while SCIN-overexpression 
group had decreasing trend of mesenchymal markers (Figure 5e-f). These results indicated SCIN functioned as a tumor suppressor by regulating EMT progression in GC cells.

\section{MiR-30 la-5p targeted SCIN and promoted EMT progression by activating STAT3 and NF-KB signaling.}

In the previous results, we have confirmed that high level of miR-301a-5p was related to more proliferative ability and higher motility of GC cells. Meanwhile, western blot results showed expression of EMT-related proteins altered when transfecting cells with miR-301a-5p mimics or inhibitors (data was showed before). Whereafter, we analyzed protein expression of key signaling and found NF-kB signaling was activated by inducing higher level of miR-301a-5p in GC cells. Since researchers have confirmed that continuously activated STAT3 could maintain the continuous activation of the NF-KB signaling pathway in tumors[18], we tested STAT3 and NF-kB as well as their active forms of phosphorylation (Figure 6a-b). When changing the expression level of SCIN, the adjustment of activation of STAT3 and NF-KB signaling displayed the same tendency (Figure 6c-d). Our data suggest that miR-301a-5p acts as an oncogene by suppressing the expression of SCIN and regulating gastric cancer progression via STAT3 and NF-kB signaling.

\section{Discussion}

Accruing data has shown that miR-301a-5p played momentous roles in tumor progression as tumor suppressor or oncogene $[7,8,19,20]$. MiR-301a was verified upregulated in GC and associated with progression and poor prognosis[21]. In the present study, we found that miR-301a-5p significantly increased in both GC tissues and cell lines. Also, it promoted GC cell proliferation, migration and invasion ability in vitro. Furthermore, SCIN was confirmed as a functional target of miR-301a-5p.
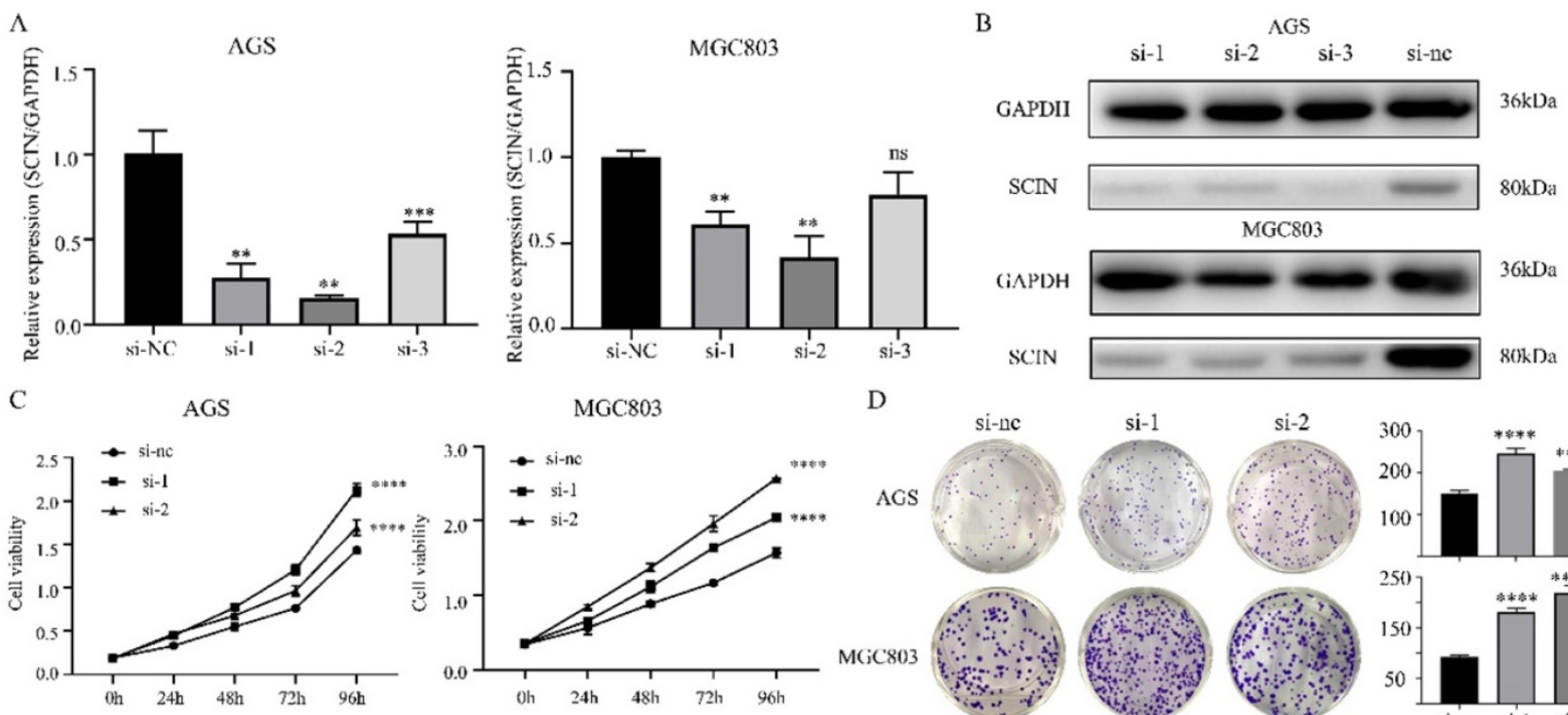

D
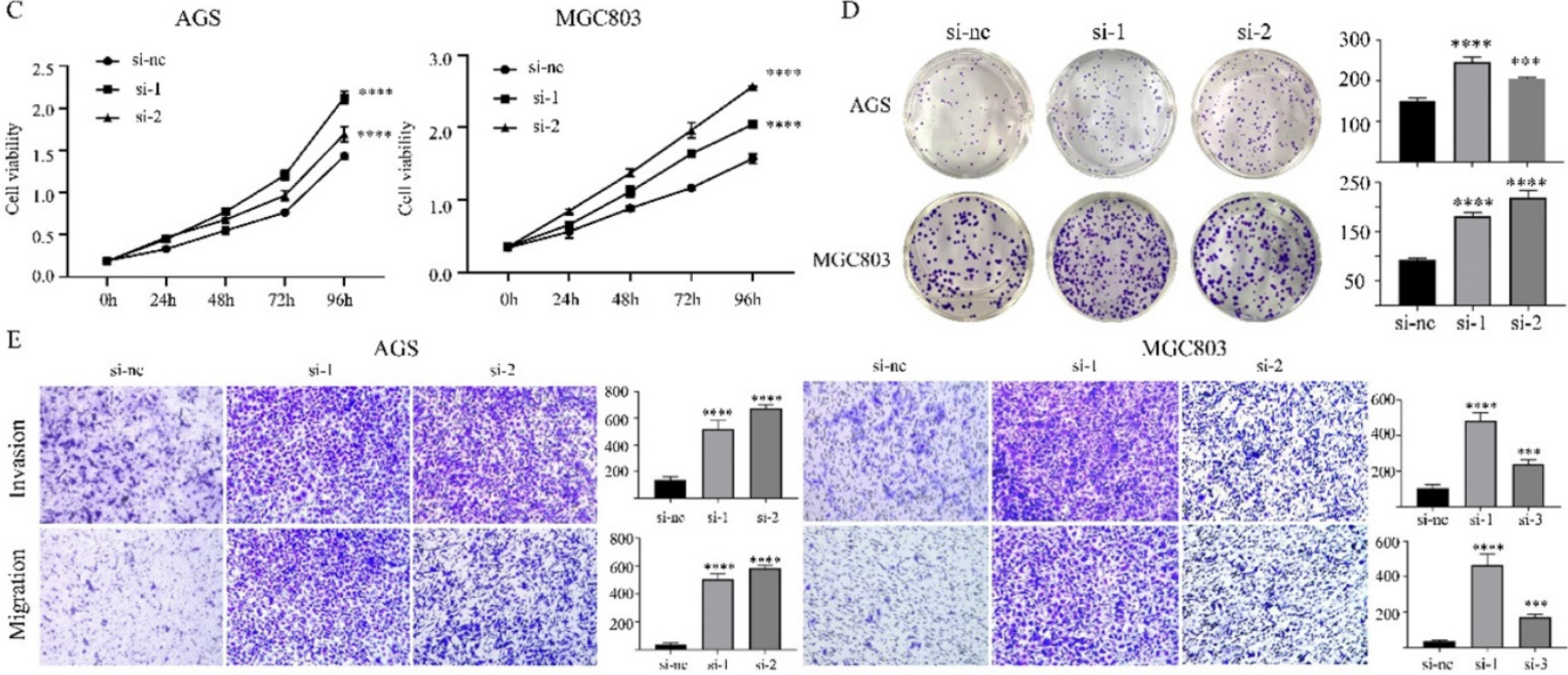

$\mathrm{MGC} 803$

si-2

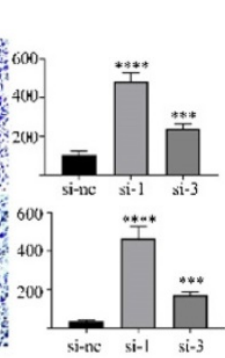

Figure 4: Down-regulation of SCIN promotes GC cells proliferation, migration and invasion in vitro. (a-b) Knockdown efficiency of SCIN in AGS and MGC803 was confirmed by qPCR and western blot. (c-d) Cell proliferation was determined by CCK-8 and colony formation assays after transfected with SCIN siRNA. (e) Cell migration and invasion were assessed by Transwell assays after transfection with SCIN siRNA (magnification, $40 \times$ ). ${ }^{*} \mathrm{p}<0.05,{ }^{* *} \mathrm{p}<0.01,{ }^{* * *} \mathrm{p}<0.001, * * * * \mathrm{p}<0.0001$. 
A

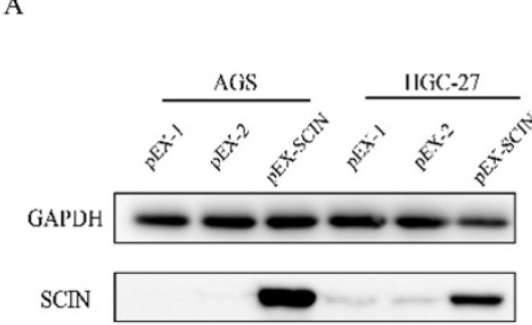

C

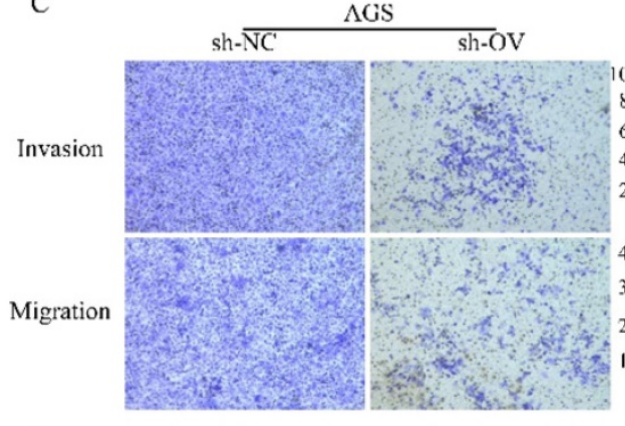

D

E

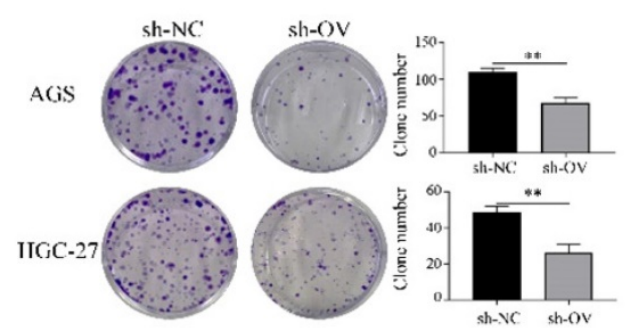

B $\quad 0.87 \quad$ AGS

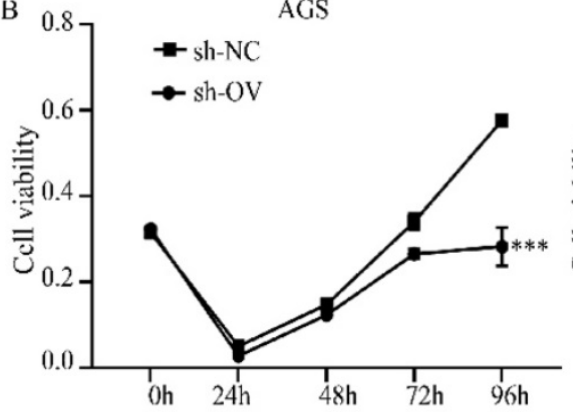

HGC-27

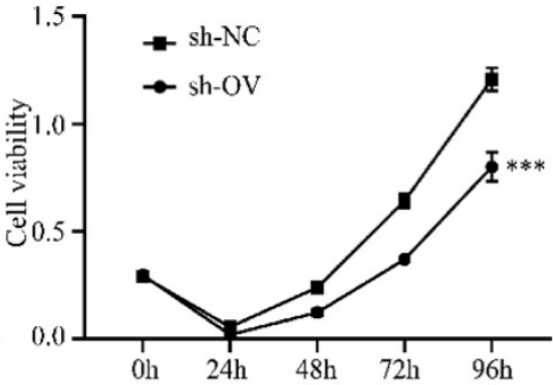

HGC-27 sh-OV

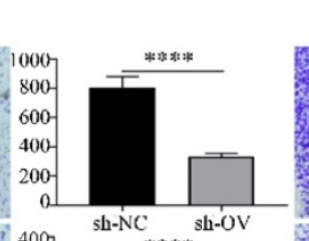

sh- $\overline{\mathrm{NC}}$
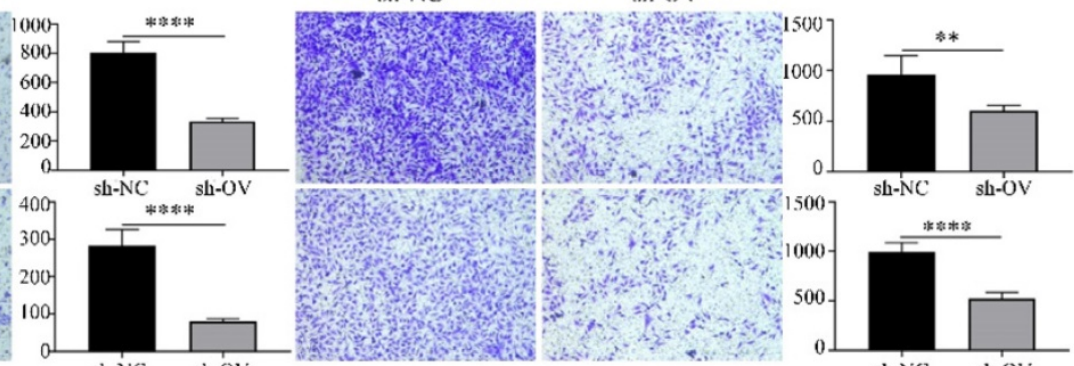

$$
\text { sh-.NC sh-OV }
$$
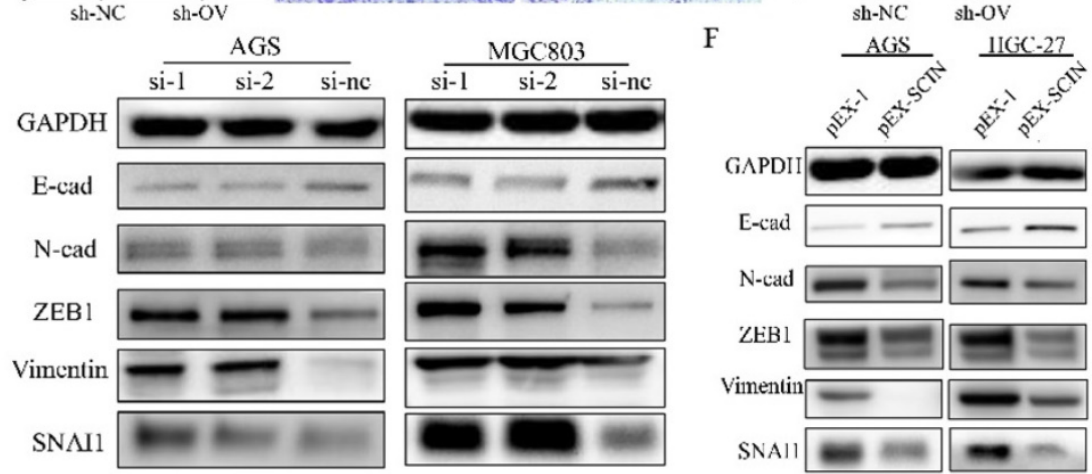

Figure 5: Overexpression of SCIN reduces GC cell proliferation, migration and invasion ability in vitro. (a) Overexpression efficiency of SCIN in $\mathrm{HGC}-27$ and MGC803 was confirmed by western blot. (b, d) Cell proliferation was determined by CCK-8 and colony formation assays after transfected with SCIN sh-RNA. (c) Cell migration and invasion were assessed by Transwell assays after transfection with SCIN sh-RNA (magnification, 40×). (e-f) EMT markers in GC cell were measured after transfection with SCIN siRNA or sh-RNA. * $p<0.05, * * p<0.01, * * * p<0.001, * * * * p<0.0001$.
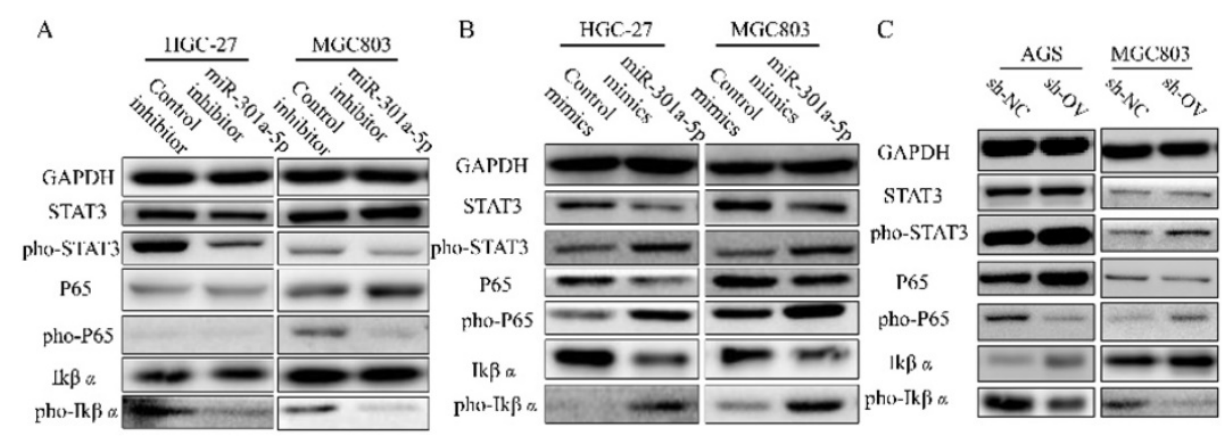

$\mathrm{D}$

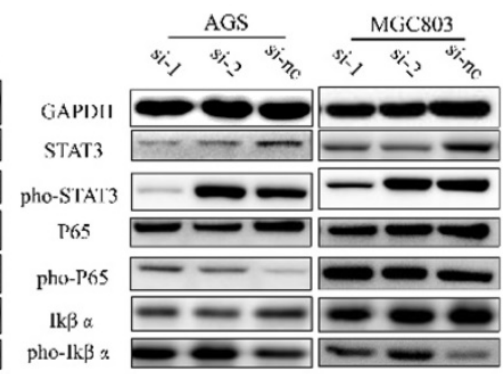

Figure 6: STAT3 and NF-KB signaling are activated by overexpressing miR-301a-5p or down-regulating SCIN. (a-b) STAT3 and NF-KB signaling-related proteins which were extracted from HGC-27 and MGC803 after transfection with mimic or inhibitor of miR301 a-5p were measured by western blot assay. (c-d) STAT3 and NF-KB signaling-related proteins which were extracted from AGS and MGC803 after overexpression or knockdown of SCIN were measured by western blot.

MicroRNAs is a category of short, non-coding RNA with about 22 nucleotides which could act as mRNA sponge and suppress target gene translation or inducing its degradation[4]. What's more, microRNAs are involved in various pathogenesis including cancer and play central roles in competing endogenous RNAs (ceRNAs) network[22]. Thus, it's acceptable for us to hypothesize that miRNAs might be a potential diagnostic marker and promising therapeutic target in cancers. In the present study, we validated that miR-301a-5p was upregulated in gastric cancer tissues and cell lines and the clinical data 
showed that increasing of miR-301a-5p was significantly correlated with advanced TNM stage and tumor size, suggesting that miR-301a-5p might be involved in GC progression. Also, Kaplan-Meier analysis showed that high expression level of miR-301a-5p was correlated with shorter overall survival of patients. Moreover, we found interfering with the expression of miR-301a-5p dramatically inhibited proliferation, migration and invasion ability in HGC-27 and MGC803 cells, while overexpression of miR-301a-5p promoted proliferation, migration and invasion in MGC803 and AGS. These data implicated miR-301a-5p acted as oncogene in gastric cancer.

Non-coding RNAs can be divided into several types based on length. MiRNAs are single-stranded RNAs and play as the negative regulators of their targeted mRNAs by based pairing to $3^{\prime}$-UTR and then targeted genes undergo translation block or mRNAs decay[23-25]. In the present study, we used bioinformatics analysis and screened out that SCIN had a complementary sequence to miR-301a-5p in its 3'-UTR. Then, two methods were used to confirm SCIN was the target of miR-301a-5p. Firstly, dual-luciferase reporter assay was performed on HEK-293T cell. And the results showed that miR-301a-5p significantly repressed the luciferase activity in cells transfected with vector carrying wild-type SCIN 3'-UTR but not inhibited the luciferase activity transfected with vector carrying mutation sites. Secondly, ectopic expression of miR-301a-5p in GC cells was performed and the expression of miR-301a-5p was verified by qRT-PCR, meanwhile, we tested the SCIN mRNA and protein expression level and found it was decreased. These results both indicated that high miR-301a-5p and low SCIN expression were found in GC and they may together play significant roles in GC progression.

Although the role of SCIN in cancer has been reported before, the correlation between miR-301a-5p and SCIN are unclear. In the current study, we revealed SCIN was repressed by miR-301a-5p and its repression promoted cell growth and motility in GC cells via activating the STAT3 and NF- $\mathrm{kB}$ signaling. Furthermore, the mechanistic interaction between miR-301a-5p and SCIN in regulation of gastric cancer cell phenotype was revealed.

STAT3 and NF-kB are two considerable factors controlling the ability of tumorigenesis and malignant transformation including anti-apoptosis, tumor angiogenesis and invasiveness[26, 27]. Activation and interaction of STAT3 and NF-kB signaling in cancers has been investigated extensively and STAT3 activation could prolong NK-KB continuous activation[18]. Our results showed that ectopic expression of miR-301a-5p or interference expression of SCIN in GC cells gave rise to activation of STAT3 and NF-KB signaling, and EMT markers showed mesenchymal markers expression were increased at the same time. And inhibiting miR-301a-5p or ectopic expression of SCIN presented decreasing expression of mesenchymal markers.

\section{Conclusion}

Our study found that miR-301a-5p was upregulated in GC and significantly associated with advanced tumor stage. Furthermore, we found that miR-301a-5 $p$ functioned as sponge and targeted to 3'-UTR of SCIN mRNA, and promoted the proliferation and motility of GC cell. EMT markers changed with miR-301a-5p, and increasing expression of miR-301a-5p made the EMT process tend to develop in the direction of mesenchymal transition. Additionally, miR-301a-5p mediated GC cell phenotype through regulating the activity of STAT3 and NF-KB pathway. Our findings may provide a potential diagnostic and promising candidates for GC therapeutic strategies.

\section{Acknowledgements}

This work was supported by project of the regional diagnosis and treatment center of the Health Planning Committee (No. JBZX-201903).

\section{Competing Interests}

The authors have declared that no competing interest exists.

\section{References}

1. Bray F, Ferlay J, Soerjomataram I, Siegel RL, Torre LA, Jemal A. Global cancer statistics 2018: GLOBOCAN estimates of incidence and mortality worldwide for 36 cancers in 185 countries. CA: a cancer journal for clinicians. 2018; 68: 394-424.

2. Feng RM, Zong YN, Cao SM, Xu RH. Current cancer situation in China: good or bad news from the 2018 Global Cancer Statistics? Cancer Commun (Lond). 2019; 39: 22

3. Lambert R, Saito H, Lucas E, Sankaranarayanan R. Survival from digestive cancer in emerging countries in Asia and Africa. Eur J Gastroenterol Hepatol. 2012; 24: 605-12.

4. Kim VN. MicroRNA biogenesis: coordinated cropping and dicing. Nature Reviews Molecular Cell Biology. 2005; 6: 376-85.

5. Zhu JH, De Mello RA, Yan QL, Wang JW, Chen Y, Ye QH, et al. MiR-139-5p/SLC7A11 inhibits the proliferation, invasion and metastasis of pancreatic carcinoma via PI3K/Akt signaling pathway. Biochim Biophys Acta Mol Basis Dis. 2020; 1866: 165747.

6. Zheng H, Liu JY, Song FJ, Chen KX. Advances in circulating microRNAs as diagnostic and prognostic markers for ovarian cancer. Cancer Biol Med. 2013; 10: $123-30$.

7. Wang YG, Wang T, Shi M, Zhai B. Long noncoding RNA EPB41L4A-AS2 inhibits hepatocellular carcinoma development by sponging miR-301a-5p and targeting FOXL1. J Exp Clin Cancer Res. 2019; 38: 153.

8. Wang J, Li H, Qiu S, Dong Z, Xiang X, Zhang D. MBD2 upregulates miR-301a-5p to induce kidney cell apoptosis during vancomycin-induced AKI. Cell Death Dis. 2017; 8: e3120.

9. Wang $X$, Luo G, Zhang $K$, Cao J, Huang C, Jiang $T$, et al. Hypoxic Tumor-Derived Exosomal miR-301a Mediates M2 Macrophage Polarization via PTEN/PI3Kgamma to Promote Pancreatic Cancer Metastasis. Cancer Res. 2018; 78: 4586-98.

10. Lu Z, Li Y, Takwi A, Li B, Zhang J, Conklin DJ, et al. miR-301a as an NF-kappaB activator in pancreatic cancer cells. EMBO J. 2011; 30: 57-67.

11. Li X, Li J, Cai Y, Peng S, Wang J, Xiao Z, et al. Hyperglycaemia-induced miR-301a promotes cell proliferation by repressing p21 and Smad4 in prostate cancer. Cancer Lett. 2018; 418: 211-20. 
12. Zheng JZ, Huang YN, Yao L, Liu YR, Liu S, Hu X, et al. Elevated miR-301a expression indicates a poor prognosis for breast cancer patients. Sci Rep. 2018; 8: 2225.

13. He C, Yu T, Shi Y, Ma C, Yang W, Fang L, et al. MicroRNA 301A Promotes Intestinal Inflammation and Colitis-Associated Cancer Development by Inhibiting BTG1. Gastroenterology. 2017; 152: 1434-48 e15.

14. Zunino R, Li O, Rose SD, Romero-Benitez MM, Lejen $\mathrm{T}$, Brandan $\mathrm{NC}$, et al. Expression of scinderin in megakaryoblastic leukemia cells induces differentiation, maturation, and apoptosis with release of plateletlike particles and inhibits proliferation and tumorigenesis. Blood. 2001; 98: 2210-9.

15. Vaysse A, Fang S, Brossard M, Wei Q, Chen WV, Mohamdi H, et al. A comprehensive genome-wide analysis of melanoma Breslow thickness identifies interaction between CDC42 and SCIN genetic variants. Int J Cancer. 2016; 139: 2012-20.

16. Miura N, Takemori N, Kikugawa T, Tanji N, Higashiyama S, Yokoyama M. Adseverin: a novel cisplatin-resistant marker in the human bladder cancer cell line HT1376 identified by quantitative proteomic analysis. Mol Oncol. 2012; 6: 311-22.

17. Zhang ZH, Zhang W, Zhou JD, Zhang TJ, Ma JC, Xu ZJ, et al. Decreased SCIN expression, associated with promoter methylation, is a valuable predictor for prognosis in acute myeloid leukemia. Mol Carcinog. 2018; 57: 735-44

18. Lee H, Herrmann A, Deng JH, Kujawski M, Niu G, Li Z, et al. Persistently activated Stat 3 maintains constitutive NF-kappaB activity in tumors. Cancer Cell. 2009; 15: 283-93.

19. Li X, Zhong M, Wang J, Wang L, Lin Z, Cao Z, et al. miR-301a promotes lung tumorigenesis by suppressing Runx3. Molecular cancer. 2019; 18(1):99.

20. Zhong $M$, Huang Z, Wang L, Lin Z, Cao Z, Li X, et al. Malignant Transformation of Human Bronchial Epithelial Cells Induced by Arsenic through STAT3/miR-301a/SMAD4 Loop. Scientific Reports. 2018; 8(1):13291.

21. Xu XD, He XJ, Tao HQ, Zhang W, Wang YY, Ye ZY, et al. Abnormal expression of miR-301a in gastric cancer associated with progression and poor prognosis. Journal of surgical oncology. 2013; 108: 197-202.

22. Qi X, Zhang DH, Wu N, Xiao JH, Wang X, Ma W. ceRNA in cancer: possible functions and clinical implications. J Med Genet. 2015; 52: 710-8

23. Ha M, Kim VN. Regulation of microRNA biogenesis. Nature reviews Molecular cell biology. 2014; 15: 509-24.

24. Jonas S, Izaurralde E. Towards a molecular understanding of microRNA-mediated gene silencing. Nature reviews Genetics. 2015; 16: 421-33.

25. Hausser J, Zavolan M. Identification and consequences of miRNA-target interactions--beyond repression of gene expression. Nature reviews Genetics. 2014; 15: 599-612.

26. Takahashi H, Ogata H, Nishigaki R, Broide DH, Karin M. Tobacco smoke promotes lung tumorigenesis by triggering IKKbeta- and JNK1-dependent inflammation. Cancer Cell. 2010; 17: 89-97.

27. Matsumura Y, Kobayashi T, Ichiyama K, Yoshida R, Hashimoto M, Takimoto $\mathrm{T}$, et al. Selective expansion of foxp3-positive regulatory $\mathrm{T}$ cells and immunosuppression by suppressors of cytokine signaling 3-deficient dendritic cells. Journal of immunology (Baltimore, Md : 1950). 2007; 179: 2170-9. 